\title{
Rowing Injuries and Related Factors in Competitive Rowing
}

\author{
Dilani Perera ${ }^{1}$, Anoja Ariyasinghe ${ }^{2}$ \\ ${ }^{1}$ Department of Physiotherapy, Faculty of Allied Health Sciences, General Sir John Kotelawala Defence University, Sri Lanka. \\ ${ }^{2}$ Department of Physiology, Faculty of Medicine, University of Peradeniya, Sri Lanka.
}

\begin{abstract}
Background: Rowing is a popular sport enjoyed at competitive levels and at recreational levels either on the water or with ergometer machines. As with any sport, extensive training results in injury. Objective: The objective of this study was to investigate the injury pattern and its causative factors among professional rowers in Sri Lanka in view to develop strategies to prevent further occurrence. Methods: A descriptive cross sectional study was conducted in the competitive period with 46 professional rowers in the Sri Lanka army in the age range of 20-33 years. An interviewer administered questionnaire was used to obtain information of demographic data, injury patterns and rowing techniques. Results: The prevalence of injury in male and female rowers was $68.8 \%$ and $57.1 \%$ respectively. The type of injury observed for female and male rowers were lower back pain (21.4\%, 37.5\%), knee pain (14.2\%, 12.5\%), pain in hand and wrist $(7.1 \%, 3.1 \%)$, hip pain $(14.2 \%, 0.0 \%)$, shoulder pain $(0.0 \%, 12.5 \%)$, ankle pain $(0.0 \%$, 3.1\%) and chest pain $(7.1 \%, 12.5 \%)$ respectively. The mean value for training volume of indoor and water training were 12 hours and 15 hours per week respectively. Both Sculling and sweep rowing techniques correlated significantly with certain injury sites for both male and female rowers. Conclusion: The prevalence of injury was significant in the Sri Lankan rowers. The anatomical sites most commonly affected were the lumbar spine and the knees. Injury is also directly related to the rowing techniques.
\end{abstract}

Keywords: rowing injuries, sweep rowing, sculling rowing, training volume

\section{Introduction}

The sport of rowing has been increasing in popularity at competitive and recreational levels through the decades [1]. This article reviews the most common rowing injuries, the associated factors such as different rowing techniques and training volume along with a description of the mechanics of the rowing stroke. This information is necessary in order to make an accurate diagnosis and the treatment protocol.

It has been reported that rowers are at a risk of developing several types of injuries including lower back, ribs, shoulder, wrist and knee problems during training and competition [13]. The commonest injury encounted in rowers is low back pain (LBP)[4-12] followed by knee injuries [8]. It has been noted that rowers have a relatively high frequency (6.1\% to $22.6 \%$ ) of rib stress fractures compared to the general population $[5,13]$. The prevalence and incidence of wrist injuries have been shown to be high in wrist loading focused sports such as rowing [14] while wrists and hand injuries are common in outdoor rowing [2]. Shoulder pain is also quite common in rowers due to repetitive overload, over-reaching at catch and due to mechanical injury [1] but the literature on this is limited. The patterns of injury are unique in the sport of rowing because of the biomechanics applied in the rowing stroke [3]. It involves a continuous repetitive motion which lays stress on various anatomical areas depending on the rowing stroke phases [2,3]. Injuries are primarily overuse $[2,8,15]$ due to change in training volume, alteration of rowing techniques and the boat $[2,3,16]$. Back pain may present differently depending on sweep versus sculling while rowers who use both sweep and sculling may lead to the development of wrist injuries [3]. It has been shown that modification in the position in rowing ergometer may lead to knee injuries [3,17]. Therefore it is essential to evaluate the different rowing techniques in order to develop protocols to prevent and treat rowing injuries [2,3].
Although, many studies have been carried out on rowing injuries in internationally, no information is available about the rowing injuries in Sri Lankan rowers. Therefore, the aim of the present study was to assess the prevalence of injuries in rowers and its associated factors in view to prevent further injuries from occurring. With a better understanding of the volume of training, mechanism of rowing, the equipments and the training procedures used, this information could aid in planning and modification of training programs and competitive tactics. This would help to develop the sport of rowing in Sri Lanka to an internationally recognized level in the future.

\section{Materials and Methods}

Subjects:

A cross sectional descriptive study was conducted to assess the pattern of injury in 46 professional rowers (32 males and 14 females) in the Sri Lanka army in the age range of 20-33 years. Written informed consent was obtained prior to testing. Ethical clearance was obtained from the Ethics Review Committee, Faculty of Medicine, University of Peradeniya, Sri Lanka (2014/EC/45).

\section{Data collection:}

Questionnaire:

An interviewer administered questionnaire was used to obtain information of demographic data, injury patterns, training volume and rowing techniques in rowers.

\section{Statistical analysis:}

The SPSS 17 version for Windows statistical software package was used to compute and report the data. Descriptive statistics were used to describe and summarize the data. Multiple regressions were used to identify the 


\section{International Journal of Science and Research (IJSR) \\ ISSN (Online): 2319-7064}

Index Copernicus Value (2013): 6.14 | Impact Factor (2015): 6.391

correlation between injury patterns and physical fitness characteristics and rowing techniques. $p$ value of less than $0.05(\mathrm{p}<0.05)$ was considered as a significance association.

\section{Results}

In total 32 male and 14 female rowers in the Sri Lankan army team participated in this study. The mean age of male and female rowers was $23.7+3.03$ and $23.43+2.10$ respectively. The prevalence of injury in male and female rowers was $68.8 \%$ and $57.1 \%$ respectively. The type of injury observed for female and male rowers were lower back pain $(21.4 \%, 37.5 \%)$, knee pain $(14.2 \%, 12.5 \%)$, hand and wrist pain $(7.1 \%, 3.1 \%)$, hip pain $(14.2 \%, 0.0 \%)$, shoulder pain $(0.0 \%, 12.5 \%)$, ankle pain $(0.0 \%, 3.1 \%)$ and chest pain $(7.1 \%, 12.5 \%)$ respectively (Figure 1$)$.

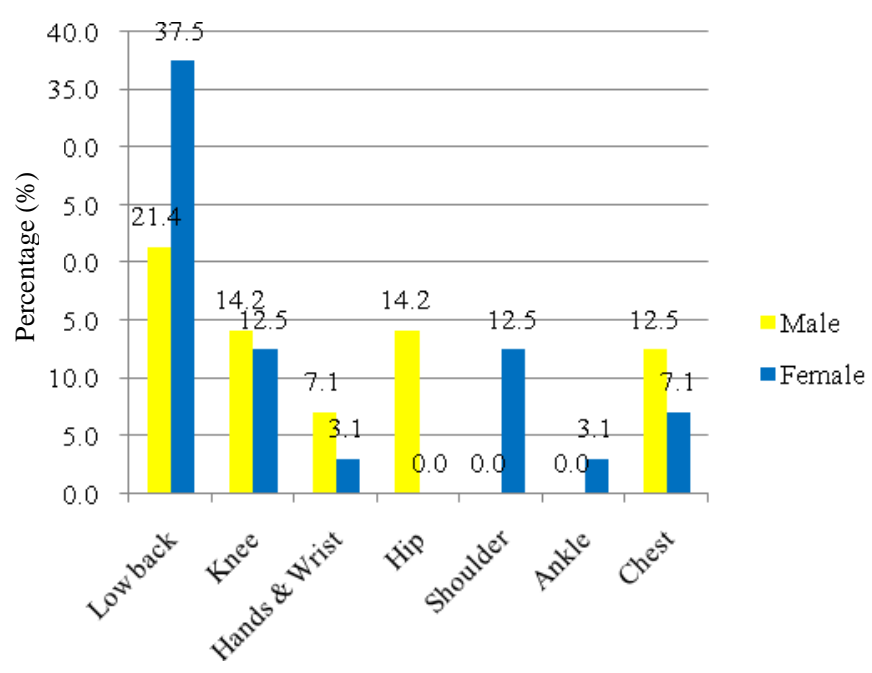

Figure 1: Prevalence of injury among male and female rowers

Figure 2 shows the different rowing techniques used by male and female rowers; sculling (44.5\%, 35.7\%), sweep (37.4\%, $14.2 \%)$ and both sculling and sweep (18.5\%, 50\%) rowing techniques respectively.

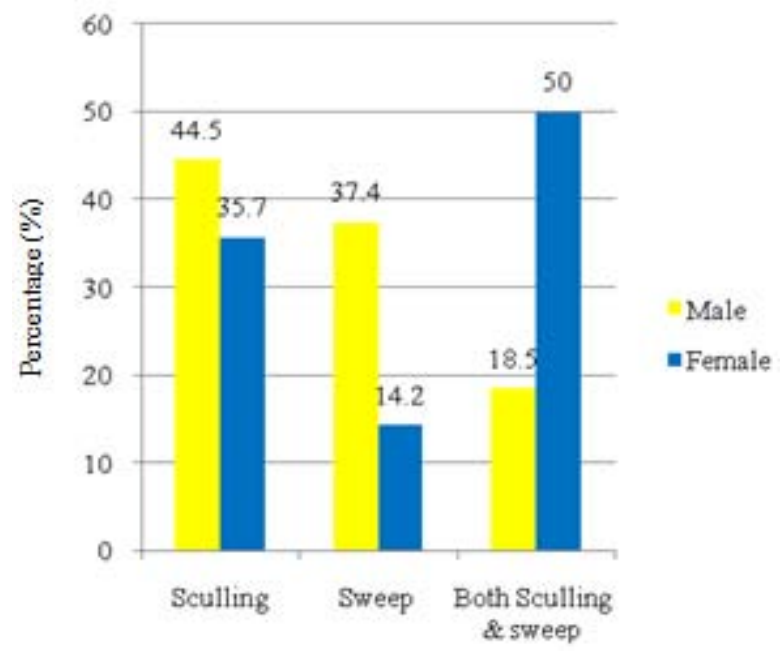

Figure 2: Rowing techniques among male and female rowers
The relationship between different rowing techniques and injury patterns are shown in Table 1 . Sculling technique correlated significantly with low back and hand /wrist injury among female rowers and ankle injury among male rowers. Sweep technique correlated significantly with low back, knee, hands/wrists, hip and chest injuries among female rowers $(\mathrm{p}<0.01)$, but it is not significant among male rowers. Both Sculling and sweep rowing techniques correlated significantly with knee, hip and chest injury among female rowers and knee and ankle injuries among male rowers $(\mathrm{p}<0.01)$. The volume of training correlated significantly with low back injury among female rowers and low back, knee and chest injuries among male rowers $(\mathrm{p}<0.01)$.

Table 1: Association between pattern of injury with sculling, sweep and both sculling \& sweep rowing technique

\begin{tabular}{|c|c|c|c|c|c|c|}
\hline $\begin{array}{c}\text { Pattern of } \\
\text { injury }\end{array}$ & \multicolumn{2}{|c|}{$\begin{array}{c}\text { Sculling rowing } \\
\text { P value }\end{array}$} & \multicolumn{2}{|c|}{$\begin{array}{c}\text { Sweep rowing } \\
P \text { value }\end{array}$} & \multicolumn{2}{|c|}{$\begin{array}{c}\text { Both Sculling \& } \\
\text { sweep rowing } P \\
\text { value }\end{array}$} \\
\cline { 2 - 7 } & Male & Female & Male & Female & Male & Female \\
\hline $\begin{array}{c}\text { Lower } \\
\text { back }\end{array}$ & 0.17 & $0.00^{* *}$ & 0.55 & $0.00^{* *}$ & 0.50 & 0.29 \\
\hline Knee & 0.25 & 0.40 & 0.09 & $0.00^{* *}$ & $0.00^{* *}$ & $0.00^{* *}$ \\
\hline $\begin{array}{c}\text { Hands \& } \\
\text { wrist }\end{array}$ & - & $0.00^{* *}$ & - & $0.00^{* *}$ & 0.25 & 0.43 \\
\hline Hip & & 0.20 & & $0.00^{* *}$ & & $0.00^{* *}$ \\
\hline Shoulder & - & - & - & - & 0.50 & - \\
\hline Ankle & $0.00^{* *}$ & - & 0.09 & - & $0.00^{* *}$ & - \\
\hline Chest & 0.08 & 0.20 & 0.09 & $0.00^{* *}$ & 0.50 & $0.00^{* *}$ \\
\hline
\end{tabular}

${ }^{*} \mathrm{p}<0.05$ - significant

$* * \mathrm{p}<0.01$-highy significant

\section{Discussion}

The injury incidence is directly related to the volume of training and different rowing techniques. The rowing injuries are primarily due to overuse [18]. A high percentage of rowers in this study suffered from low back pain followed by knee pain (Figure 1). Several past studies show that low back pain is a common complaint in the rowing population $[2,4,8,11,15,17,19]$ and it is prevalent in almost all rowers undergoing serious rowing training [9] with ergometer pieces longer than 30 minutes [11].

In the present study it was observed that female have a higher prevalence of low back pain than male rowers. Similarly, Howell's revealed an $82.2 \%$ incidence of low back pain among elite lightweight female rowers compared with an age- and sex-matched general population which was $20 \%$ to $30 \%$ [20]. In the present study both sculling and sweep rowing techniques significantly correlated with low back pain in both male and female rowers. The flexion and compressive loading during each stroke are similar for sculling and sweep ergometer rowing [3]. In addition to flexion and compression, sweep rowers rotate the trunk during rowing ergometer [22]. These forces can cause injury to the lumbar spine [22]. Sweep rowers who change sides during a season also had a significantly greater chance of developing low back pain than those who did not change the sides $[3,8]$. The back behaves as a braced cantilever during the rowing stroke and transfers the power from the legs to the oar. Therefore it is suggested that evaluation of the load 


\section{International Journal of Science and Research (IJSR) \\ ISSN (Online): 2319-7064}

Index Copernicus Value (2013): 6.14 | Impact Factor (2015): 6.391

at the oar while maintaining a steady pace and changing the stroke rate showed a significant decrease in load [3].

Second most common injury among Sri Lankan rowers was knee injuries. Males suffered from knee pain more than females. But Kristine and Karlson had shown that this is more common among women than men [2]. Knee is a commonly affected site for injury and it could be due to chondromalacia patella and Ilio tibial band (ITB) friction syndrome [15]. Rowing involves extreme loading of the patella-femoral joint [3]. This is because rowing training is designed to increase the strength of the quadriceps muscle which can cause significant patella pressure. Chondromalacia patella can present as anterior knee pain during rowing or quadriceps strengthening training activities. It is also commonly found in those with genu varus [2,3]. The ITB friction syndrome is also fairly common in the rowing population. ITB band may produce inflammation over the lateral femoral condyle resulting in localized pain [2,3]. Knee injuries are common among Sri Lankan rowers who perform both sculling and sweep rowing techniques and it showed significant correlation. It is suggested that several modifications in the position in rowing ergometre can alter the patellofemoral mechanism; as an example, raising the height of the foot stretcher decreases the knee flexion angle leading to a decrease in patella compression [3]. Functional knee varus can be addressed by changing the rotation of the toe-in or toe-out position of the sneakers. Front or back stops can be placed on the track of the sliding seat of the shell or ergometer to limit the arc for knee flexion or extension [17].

Wrist and hand injuries are also common in both male and female rowers in the present study. These injuries are common in outdoor rowing as feathering the oar is an unused activity but it is not used in indoor rowing ergometer training [2]. This injury is directly related to the rowing techniques. Wrist and hand injuries were common in both sweep and sculling techniques in the present study. The sweep rowers use one hand to rotate (feather) the oar, while the sculling rowers have to use both hands [3]. In sweep rowers, this constant motion may lead to the development of crossover extensor tenosynovitis in wrist [3] which is caused by compression of the radial extensor tendons [23]. The key to prevention of extensor tenosynovitis is to keep the hand and wrist warm by using long sleeves and commercially available fleece covers that protect the hand and wrist while rowing outdoors.

Chest injuries are common among both male and female rowers in the present study. The majority of these injuries are rib stress fractures which make up with a relatively high frequency values $(6.1 \%$ to $22.6 \%)[, 5,13,15,17,20]$. In the present study; training volume correlated significantly with chest injuries in male rowers $(\mathrm{p}<0.05)$ while chest injuries significantly correlated with the sweep rowing technique and both sculling and sweep rowing techniques in female rowers. Rib stress fractures generally occur when the rowers are involved in long periods of rowing ergometre training. Biomechanical analysis of the ribs predicts that the bending moment occurs at the middle third of the rib in its posterolateral segment [2]. Rib stress fractures generally occur during periods of intense training with a relatively low stroke rate and high load per stroke. This occurs during the fall and winter training months when the rowers are doing long aerobic pieces on the rowing ergometer in temperate countries. Chest injuries are significantly correlated with the sweep rowing technique and both sculling and sweep rowing techniques $(\mathrm{p}<0.01)$ in female rowers.

\section{Conclusion}

In conclusion, the prevalence of injuries among both male and female rowers in the Sri Lankan army was significant. The anatomical sites most commonly affected were the lumbar spine and the knees. The present study emphasizes the importance of being aware of the injury patterns, different rowing techniques and biomechanical forces affecting the athlete so that measures could be initiated to prevent disability and enhance the performance of rowers in the future.

\section{Acknowledgement}

The authors would like to acknowledge all members of the Sri Lankan army rowing teams, team coach and in charge of rowing for their support and collaboration during the study.

\section{References}

[1] Rumball J, Lebrun CM, DiCiacca SR, Orlando K. Rowing Injuries. Sports Medicine 2005; 35(6):537-555.

[2] Karlson KA. Rowing injuries: identifying and treating musculoskeletal and non musculoskeletal conditions. Journal of Sport Medicine and Physical fitness 2000; 28(4):40.

[3] Hosea TM, and Hannafin, JA, rowing injuries, Sports Health 2012; 4(3): 236-245.

[4] Boland Al, Hosea TM. Rowing and sculling and the older athlete. Clinics in Sports Medicine 1991;10(2):245- 256.

[5] Hickey GJ, Fricker PA, McDonald WA. Injuries to elite rowers over a 10-yr period. Medicine and Science in Sports and Exercise 1997;29:1567-1572.

[6] Howell D. Musculoskeletal profile and incidence of musculoskeletal injuries in lightweight women rowers. American Journal of Sports Medicine 1984;12:278-281.

[7] Roy SH, De Luca CJ, Snyder-Mackler L, Emley MS, Crenshaw, RL, Lyons JP. Fatigue, recovery and low back pain in varsity rowers. Medicine and Science in Sport and Exercise 1990;22(4):463-469.

[8] Smoljanovic T, Bojanic I, Hannafin J, Hren D, Delimar D, Pecina M. Traumatic and Overuse Injuries Among International Elite Junior Rowers. American Journal of ports Medicine 2009;37(6):1193-1199.

[9] Stallard M. Bachache in oarsmen. British Journal of Sports Medicine 1980;14:105-108.

[10] Stallard M. The challenge of rowers backache. Sport and Medicine Today 1999;1:53-55.

[11] Teitz C, O'Kane J, Lind BK, Hannafin JA. Back pain in Intercollegiate rowers. American Journal of Sports Medicine 2002;30(5):674-679.

\section{Volume 5 Issue 4, April 2016


[12] Wilson F, Gissane C, Gormley J, Simms C. A 12-month prospective cohort study of injury in international rowers. British Journal of Sports Medicine 2010;44(3):207-214.

[13] Warden SJ, Gutschlag FR, Wajswelner H, Crossley KM. Aetiology of rib stress fractures in rowers. Sports Medicine 2002;32(13):819-36.

[14]Laura S Kox, P Paul F M Kuijer, Gino M M J Kerkhoffs, Mario Maas, Monique H W Frings-Dresen, Prevalence, incidence and risk factors for overuse injuries of the wrist in young athletes: a systematic review. British Journal Sports Medicine 2015 ;49:1189-1196.

[15] Hosea TM, Boland A, McCarthy K, Kennedy T. Rowing injuries. Postgrad Adv Sports Med 1984;3(9):1.

[16] Mitchell T, O'Sullivan PB, Burnett AF, Straker L, Smith A. Regional differences in lumbar spinal posture and the influence of low back pain. BMC Musculoskeletal Disorders 2008;9:152-162.

[17] Hannafin J, Hosea T. Oar sports. In: Garret WE, Kirkendall DT, Squire DL, editors. , eds. Principles and Practice of Primary Care Sports Medicine. Philadelphia, PA: Lippincott, Williams \& Wilkins; 2001:531-540.

[18] Swarup Mukherjee, Hin Fong Leong, Simin Chen, Young Xiang Wayne Foo, Hong Kiat pek, Injuries in Competitive Dragon Boating, Orthopaedic Journal of Sports Medicine 2014;2(11).

[19] Maurer M, Soder RB, Baldisserotto M. Spinal abnormalities depicted by magnetic resonance imaging in adolescent rowers. American Journal of Sport Medicine 2011;39 (2):392-397.

[20] Holden DL,Jackson DW. Stress fracture of the ribs in female rowers. American Journal of Sport Medicine 1985;13(5):342-8.

[21] Adams M, Dolan P. Recent advances in the lumbar spine mechanics and their clinical significance. Clinical Biomechanics 1995;10:13-19.

[22] Williams J. Surgical management of traumatic noninfection tenosynovitis of the wrist extensors. Journal of Bone of Joint Surgery 1977;59:408.

[23] Karlson KA. Rib stress fractures in elite rowers: a case series and proposed mechanism. American Journal of Sport Medicine 1998;26(4):516. 